P. Reichenbach, U. Georgi, U. Oertel, T. Kämpfe, B. Nitzsche, B. Voit, and L. M. Eng

\title{
6 Optical antennae for near-field induced nonlinear photochemical reactions of photolabile azo- and amine groups
}

\begin{abstract}
We performed a series of nonlinear-optical, photochemically-induced experiments using different copolymers which were UV-sensitized at around $400 \mathrm{~nm}$ through various chemical side groups (e.g. NVOC-protected amines). Our approach focuses on inducing very localized photoreactions on an area much smaller than the diffraction-limited optical spot, using nonlinear optical effects in the near-fields of metallic nanoparticle (NP) antennae embedded in the polymer thin film. We thus illuminated the copolymers with $100 \mathrm{fs}$ pulses at a $790 \mathrm{~nm}$ exciting wavelength in order to trigger photoreactions through nonlinear optical effects at around $400 \mathrm{~nm}$. Spin coating the copolymers onto both y-cut lithium niobate (LNO) sample surfaces (which is an excellent SHG amplifier) and onto bare glass surfaces (no SHG) and then comparing the as-induced nonlinear optical photoreactions showed a direct two-photon absorption to be the dominant photoreaction mechanism, while SHG radiation (as emitted by LNO) did not show any significant influence. High efficiency of this process can be achieved by the near-field enhancement close to metallic NPs present in the polymer thin film. First evidence is given here with protected amine side groups when spin coating the copolymer over sharp gold edges as obtained from triangular metallic NPs (i.e. so-called Fischer pattern); after laser exposure at $790 \mathrm{~nm}$, the photochemically deprotected amines were successfully photo-labeled and then imaged by STORM microscopy.
\end{abstract}

\subsection{Introduction}

Photolithographic structuring has developed into one of the most important tools of nanostructuring to date. The most prominent example is the nanostructuring of silicon [1]. Combining photolithographic techniques with photochemistry allows inclusion of the vast variety of chemical structuring and functionalization into the repertoire of photolithography. Examples are the photoreactions of protected amineterpolymers under UV illumination $[2,3]$ for instance. In this case the illuminated and deprotected parts of the amines are easily functionalized with fluorescence markers or other molecules for further use or monitoring purposes.

An important milestone in the research into photochemistry at the nanometer length scale is the use of metallic nanoparticles (NPs) which concentrate light in their near-fields. One example is photoreactions of resist thin films in the near-field 
of metallic nanorods [4] or bow-tie antennae [5]. Such photoresist reactions in the vicinity of the NP's near-fields were also shown to be successful when triggered via nonlinear optical absorption [6-8] rather than in the linear optical regime.

The goal of our work was thus to trigger photochemical reactions in polymer matrices on the nanometer length scale [9-12]. Furthermore, as indicated by the examples above we also intended to induce such processes in the near-fields of metallic nanoparticles. Our primary idea was to embed these metallic (e.g. gold) nanoparticles into a photosensitive polymer and illuminate them with focused $100 \mathrm{fs}$ pulses at an infrared (IR) wavelength of about $790 \mathrm{~nm}$. The localized photoreaction would then take place at around $395 \mathrm{~nm}$, profiting from the NP's plasmonic amplification.

We did not concentrate on photoresists as photosensitive material but rather developed copolymers mainly consisting of MMA and a comonomer add-on which carries the photosensitive group such as the protected amine side groups already mentioned above [2, 3]. These polymer matrices are optically transparent at $790 \mathrm{~nm}$; thus no photoreaction occurs over the whole diffraction-limited optical spot of the exciting IR beam. Instead, the polymer is photosensitive in the range of $395 \mathrm{~nm}$.

We intended to use the second harmonic radiation (SHG) from the nanoparticles to induce the photoreaction at $395 \mathrm{~nm}$ primarily, as many investigations demonstrated that metallic nanostructures with strong field enhancement must also constitute good SHG sources [13-17], and investigations in our group also comprised nanoparticle SHG $[9,18]$.

However, the question had to be solved whether SHG or multiphoton absorption processes are actually the dominant reaction mechanisms. Experiments with polymer matrices on lithium niobate single crystals (sc-LNO) as a good SHG source showed the dominance of a two-photon absorption. This two-photon absorption also profits from high absorbance at the SHG wavelength of $395 \mathrm{~nm}$, being equivalent to a single-photon absorption at this wavelength.

Metallic nanoparticles can be joined with the polymer in many ways, mostly by NP embedding (Fig. 6.1) or overcoating the NP array sitting on a substrate with a polymer thin film. As nonlinear optical processes are much stronger in the near-fields of

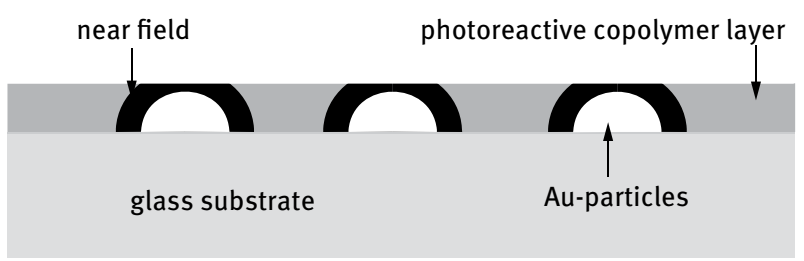

Fig. 6.1: Sketch of the principal idea of our experiments: a photoreactive copolymer layer (dark gray) is spin coated onto a glass substrate (light gray) carrying Au nanoparticles (NPs; white). Upon pulsed laser irradiation at $\sim 790 \mathrm{~nm}$, the NPs produce a considerable enhanced near-field (black zones around NPs) where the photochemical reaction concentrates to a sub-100-nm spatial area. 
the particles than in the far fields, photochemical reactions triggered by these processes can be confined to volumes in the sub-100-nm regime. The outcome of such a photochemical reaction and its localization can in turn be monitored through several different methods, depending solely on the type of chemicals used for the photolabile group as will be described below.

\subsection{Experimental design}

Our experiments were conducted with the following setup.

The femtosecond laser beam $(\sim 100 \mathrm{fs}$ at $790 \mathrm{~nm}$ at a repetition rate of $75 \mathrm{MHz}$ ) passes an upright standing $40 \times$ air objective and is focused onto the sample surface positioned via a xyz piezo stage. For each writing process the focus is moved about $7 \mu \mathrm{m}$ away from the sample surface in order to broaden the area of photoreaction and include more nanoparticles. Typically, the sample consists of a cover glass carrying a spin-coated photosensitive polymer matrix layer about $100 \mathrm{~nm}$ thick which may also include embedded metallic nanoparticles.

Using the piezo stage we are also able to photochemically "write" any arbitrary pattern onto these films: While illuminating the sample surface with the focused laser beam, the photosensitive polymer layer is scanned laterally through the focus, thus resulting in lines of a few $\mu \mathrm{m}$ in length where the photoreaction has taken place. These experiments were carried out on pure polymer substrates spin coated onto glass as well as with embedded nanoparticles.

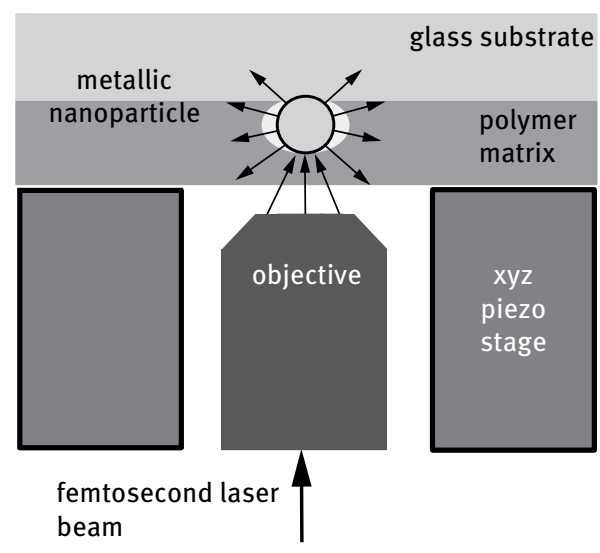

Fig. 6.2: Sketch of the experimental setup used in these studies. The sample structure (described in Fig. 6.1) is inserted upside down into the setup with the polymer film facing the objective. The femtosecond laser beam is focused onto the sample surface and excites the plasmonic NPs to produce a strong nearfield both at the fundamental and the second harmonic (SHG) field (see arrows emitted from the NP). The xyz piezo stage is used to adjust the focus to the sample surface as well as to initiate $x y$-sample structuring.

Three kinds of copolymers were used for our experiments:

(a) Copolymers with azobenzene side groups which photochemically react with a trans-cis isomerization; this process may be tracked optically through a pronounced change in absorbance [12]. 
(b) Copolymers with azosulfonate side groups which decay with subsequent crosslinking; the resulting local changes of elasticity are detectable with AFM [11, 19].

(c) Copolymers with protected amine side groups using NVOC. After photochemical separation of the NVOC molecules, different dyes such as fluorescent markers may be chemically attached to the deprotected amines, highlighting the reaction sites. This enables detection by different kinds of fluorescence microscopy [2].

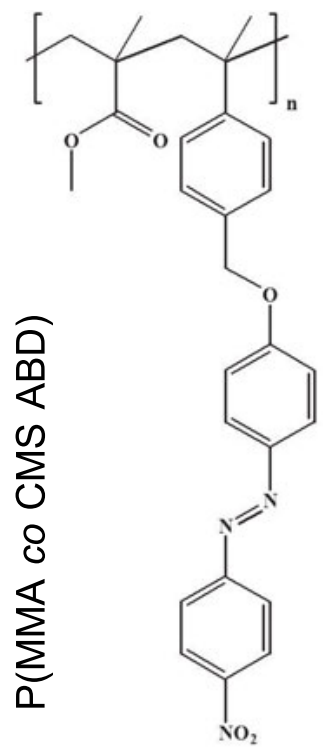

(a)

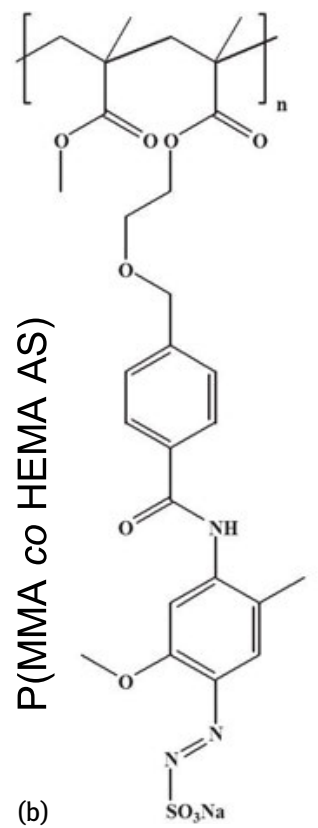

(b)

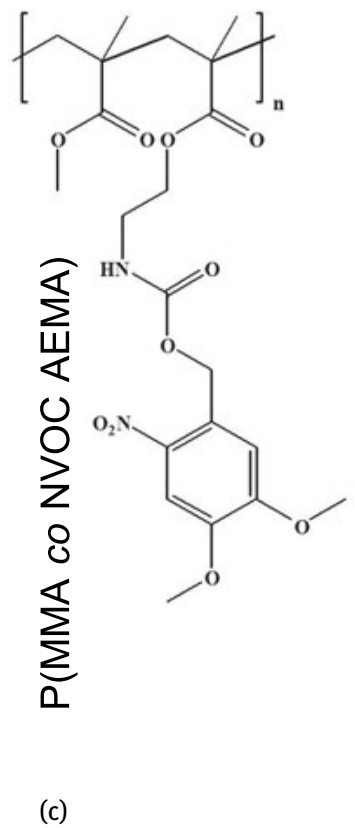

(c)

Fig. 6.3: Chemical structures of the copolymers used in this work Several different polymer sidechains were synthesized to the backbone, i.e. (a) azobenzene derivatives (ABD), (b) azosulfonates (AS), and (c) NVOC-protected amines (AEMA).

As stated in the introduction, we intended to trigger the photochemical reactions by SHG radiation from the embedded nanoparticles. However, the question arose whether or not the photoreaction may also be induced by direct two-photon absorption. To clarify the impact of these different reaction mechanisms on the localization of the photoreaction, we modeled the particle near-fields at the fundamental and second-harmonic frequency by evaluating the near-fields $\boldsymbol{E}^{\omega}$ (fundamental) and $\boldsymbol{E}^{2 \omega}$ (SHG) for a Hertzian dipole emitter. The calculated photoreaction density thus turns out to be proportional to $\left|\boldsymbol{E}^{\omega}\right|^{4}$ and $\left|\boldsymbol{E}^{2 \omega}\right|^{2}$ for the two-photon and SHG absorption processes, respectively. Hence, photoreactions triggered by a two-photon absorption process were found to be more effective and even to be much more localized than SHG-induced photoreactions. 
To find out which of the two mechanisms in our experiments is dominant we spin coated the polymer matrix both onto glass samples and onto a y-cut lithium niobate (LNO) surface without using any nanoparticles. Moving the polymer matrix in the xy plane through the femtosecond laser focus (100 fs, $790 \mathrm{~nm}$ ) then allowed the writing of several lines of a variable exposure by altering the laser power density. We then tried to observe and monitor the as-induced photochemical reactions. While lithium niobate is a strong second harmonic source (for light polarized along the extraordinary optical axes), glass does not show any SHG radiation at all, thus allowing a fair comparison of the two photoreactions.

In the subsequent experiments including embedded nanoparticles we used metallic nanostructures known as Fischer patterns (Fig. 6.4) [20]. They constitute flat hexagonally arranged, triangular gold islands. They are fabricated by evaporating gold onto the (glass) substrate surface through a mask built up by a monolayer of hexagonally dense-packed polystyrene beads with a diameter of about $600 \mathrm{~nm}$ [20-22]. The beads are then removed and the copolymer spin-coated over the antennae structures. The use of such Fischer patterns has three advantages: Firstly, Fischer patterns are prepared easily; secondly, their low height allows for good polymer spin-coating and spreading, while thirdly their tips and edges are very sharp hence promoting strong near-field enhancement due to the lightning rod effect.
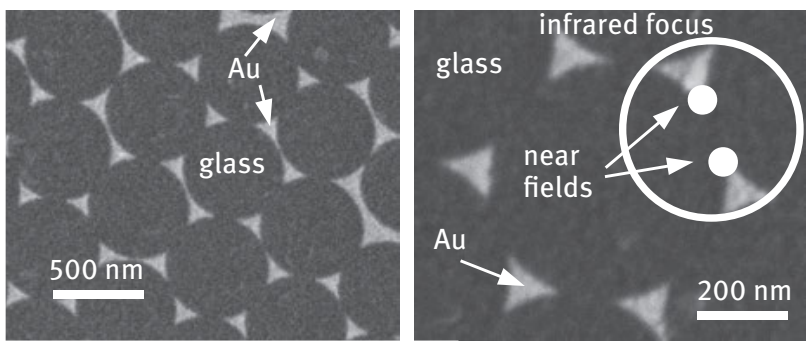

Fig. 6.4: Fischer patterns result in hexagonally arranged Au triangles grown by evaporating $\mathrm{Au}$ through a mask of close-packed polystyrene beads (not shown). The dark area in this SEM image represents the bare glass surface while the bright areas are the $20 \mathrm{~nm}$ high Au NPs. (a) SEM overview, and (b) close up with the white circle denoting the optical focus of the objective as used for excitation; note the two asperities at the triangular corners emitting the near-field at both the fundamental and SHG wavelength.

\subsection{Photochemical experiments using a copolymer carrying azobenzene side groups}

The first photosensitive polymer matrix is a PMMA-type copolymer containing an azobenzene derivative with an $\mathrm{NO}_{2}$ molecular group (Fig. 6.2 (a)) [11, 12]. Azobenzenes are already widely used for writable data carriers [23] and in volume holography 
[24]. The photochemical reaction of the azobenzene derivative selected for our work is a reversible trans-cis isomerization. This photoreaction leads to a change in absorbance of the substrate, which allows direct optical detection and monitoring. The azobenzene derivative possesses two main advantages: Firstly, it absorbs in the range of $400 \mathrm{~nm}$, while secondly the thermally induced back-reaction of the reversible trans-cis isomerization is comparably slow and proceeds over several hours [12].

The copolymer contains $80 \%$ methylmethacrylate (MMA) and $20 \%$ chloromethylstyrene (CMS) and has been synthesized using the RAFT polymerization method (reversible addition fragmentation chain transfer polymerization) [25-27] known to show minimal side reactions. The azobenzene derivative was linked covalently to the CMS parts of the copolymer [12]. The copolymer was dissolved in methylethylketone (MEK) and then spin-coated onto the glass or lithium niobate (LNO) sample surfaces to form a dense and uniform layer $70 \mathrm{~nm}$ thick. The covalent binding of the azobenzene derivative to the copolymer ensures there is no segregation of the azobenzene from the MMA/CMS-copolymer.

The photochemical experiments on glass and LNO were carried out as outlined below. The glass and LNO substrates carrying the $70 \mathrm{~nm}$ thin polymer matrix layers were inserted upside down into the measurement setup (Fig. 6.3) with the photosensitive polymer film facing the objective directly. The optical focus was adjusted to sit at the substrate sample surface. The LNO used was a y-cut crystal oriented in such a way that the linearly polarized beam yielded a maximum SHG intensity when focused onto the LNO surface. Using the piezo stage, $5 \mu \mathrm{m}$ long lines were then written on to the polymer films using different average laser powers tuned from 2 to $15 \mathrm{~mW}$. Here and in all experiments to follow, a stepwise sample motion was employed with individual steps measuring $167 \mathrm{~nm}$ in size (i.e. $5 \mu \mathrm{m}$ divided by 300 steps) and dwelling times of $50 \mathrm{~ms}$ per step.

After photochemical writing, the optical transmission of these lines was measured in-situ using the focused light of a $405 \mathrm{~nm}$ diode laser for illumination and a fiber coupling mounted above objective and sample. This in-situ measurement has the advantage that changes in the optical transmission can be monitored immediately and even before any back-reaction sets in. The $405 \mathrm{~nm}$ light was reduced to a power of a few $\mu \mathrm{W}$ before being focused through the same objective onto the sample surface, thus not inducing any further sample reaction at all. The light transmitted through the photosensitive layer was collected with an optical fiber fed into a fiber spectrometer (Maya 2000 Pro from Ocean Optics). On scanning the exposed area with the written lines through the blue laser focus we obtained images of these lines since the photoreaction considerably increased optical transmission (i.e. less absorption in the exposed areas as shown in Fig. 6.5).

As an outcome, we found that increased transmission of the lines became visible for "writing" laser power densities of $6 \mathrm{~mW}$ or above, both on the glass and LNO substrate. The absence of a significant difference between glass and LNO shows that the SHG from the LNO substrate does not significantly contribute to the photoreaction, but 

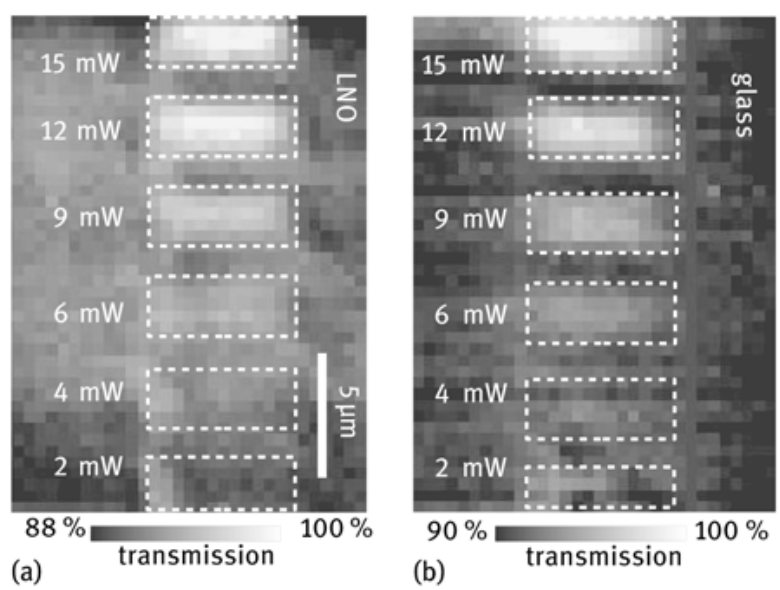

Fig. 6.5: Optically recorded transmission scans of the $70 \mathrm{~nm}$ thick azobenzene-containing copolymer spin coated onto (a) LNO and (b) glass substrates. Note the different lines (dashed frames) and their varying transmission cross-sections resulting from exposure to the focused femtosecond beam at different average power densities; notably, the higher the power, the lower the absorbance.

that the photoreaction is triggered by direct absorption of the IR light. Photochemical writing with the infrared laser radiation in cw mode and at the same power density did not result in detectable transmission changes - an indication that the photoreaction is mainly triggered by a nonlinear optical effect, namely a two-photon absorption process of the femtosecond light. ${ }^{1}$

When we compared the photon density of blue light from a monochromator used to trigger a photoreaction directly with the SHG photon density on lithium niobate, we found that the SHG photon density from the lithium niobate at $6 \mathrm{~mW}$ incident power is about three orders of magnitude lower than for the blue light from the monochromator. As SHG from nanoparticles is typically not stronger than from the y-cut LNO surface, we can expect that nanoparticle SHG also does not contribute significantly to photoreactions. Of course, SHG light is more concentrated in the particle near-fields, however, the same is the case for the near-fields at the fundamental frequency.

1 At this point the question arises whether or not the reaction might be thermally induced as well, by simple laser heating of the substrate. As cw irradiation did not trigger photoreactions, we carried out some model calculations investigating the difference in the time development of the sample temperature when hit either by pulsed or cw illumination. These calculations showed that the maximum temperature did not differ at all in both cases, which excludes sample heating as the source of the reported photoreactions. 


\subsection{Photochemical experiment with azosulfonate-carrying copolymers}

Optical detection of the photoreactions in the azobenzene-containing copolymer has a diffraction-limited resolution, so we did not carry out experiments including gold nanostructures. However, the next copolymer used was designed to change its elasticity due to the photoreaction, and such a change can be detected with AFM at a resolution of around $50 \mathrm{~nm}$. The copolymer consisted of $75 \%$ MMA and $25 \%$ HEMA (hydroxyethylmethacrylate) and was polymerized with the RAFT method. On the hydroxyl groups of the HEMA an azosulfonate group (Fig. 6.2(b)) was bound covalently in a polymer-analog reaction [12]. Again, this azosulfonate derivative was selected for its optimal absorption at $395 \mathrm{~nm}$. However, the absorption of that derivative still strongly decays between 380 and $400 \mathrm{~nm}$ and is about one order of magnitude smaller than azobenzene. The chosen azosulfonate does not isomerize reversibly but isomerization leads to a decay into $\mathrm{SO}_{3} \mathrm{Na}$ radicals and phenyl radical residuals under nitrogen evolution [28, 29]. A crosslinking reaction between the residual phenyl radicals and the polymer chains causes increased elasticity of the polymer matrix in the reaction volume, an effect which can also be used for photoresins [29]. On scanning the as-exposed area with AFM, this change of elasticity can be readily detected via phase contrast imaging. The preparation of these layers is again done by spin-coating, but this copolymer is dissolved in dimethylformamide.

This proof-of-principle was carried out by illuminating the polymer substrate through a TEM grid (Fig. 6.6(a)) using a white-light beam (UV extended spectrum) followed by AFM scans over the polymer surface. The AFM scan (Fig. 6.6 (b)) shows no topographic pattern which corresponds to the TEM grid, but the phase image (Fig. 6.6(c)) clearly shows a phase contrast pattern in accordance with exposure through the TEM grid. The phase contrast is of the order of $1^{\circ}$. Experiments including Fischer patterns should thus clearly show this phase change in the surroundings (i.e. in the near-field range) of the gold triangles.

However, before doing so we again compare the behavior of a pure polymer matrix layer on glass and on LNO. As absorbance drops strongly below $400 \mathrm{~nm}$, the exciting wavelength was shifted to $780 \mathrm{~nm}$ to achieve a two-photon wavelength of $390 \mathrm{~nm}$. The polymer substrates (70 $\mathrm{nm}$ thick) were irradiated for different laser powers and then scanned with AFM. In contrast to the azobenzene-carrying copolymer, average powers of $20 \mathrm{~mW}$ and higher had to be applied to yield lines that could be detected by AFM at all. However, these lines were not only detectable via phase contrast, but were also always accompanied by the formation of topographic swales with comparably huge depths measuring tens of $\mathrm{nm}$ in height. Similar to the azobenzenes, no differences were obtained between glass and LNO surface (indicating again the dominance of twophoton absorption), while these effects were absent for cw illumination. However, on comparing these results with the reaction of a pure PMMA layer, we found the same results even for these layers. At the high powers above $20 \mathrm{~mW}$, not only azosulfonate 

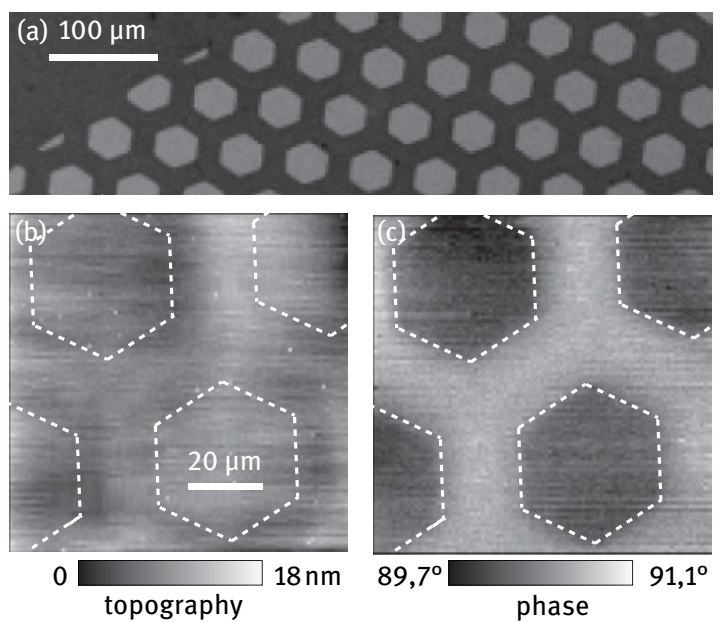

Fig. 6.6: (a) SEM image of the TEM grid structure used. (b) AFM topographic image of the azosulfonate-carrying copolymer exposed through the TEM grid using a UV-extended white light source. (c) AFM phase image of the same area showing enhanced stiffness at exposed areas (white). The border of the TEM grid structure is indicated by dashed lines in the AFM pictures for clarity.

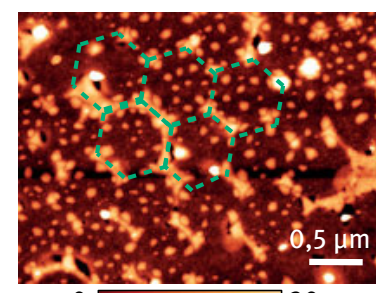

(a)

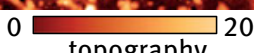

topography

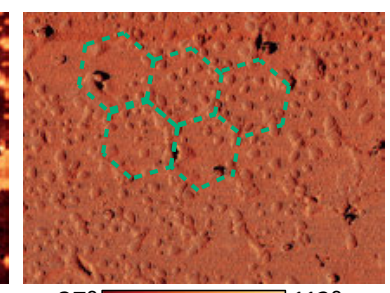

(b)

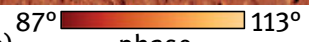

phase

Fig. 6.7: AFM topography (a) and phase (b) scans of the azosulfonate-containing copolymer spin coated over Fischer patterns. These scans were taken well inside a line that had previously been written by pulsed illumination with an average power of $0.2 \mathrm{~mW}$. We clearly differentiate these Fischer patterns in (a), marked by dashed lines, together with a fine-pitched quasi-regular arrangement of polymer debris blisters, the latter stemming from the initial exposure and insufficient dissolving of the copolymer.

decay sets in but also quite different reactions of the PMMA itself will be triggered through multiphoton absorption. This makes it difficult to detect the specific photochemical reaction of the azosulfonates unambiguously solely by AFM phase contrast.

Furthermore, the azosulfonate-carrying polymer was also spin-coated over the glass substrates carrying $10 \mathrm{~nm}$ high Fischer patterns. The topography of these triangles was also visible on the upper side of the $70 \mathrm{~nm}$ thick copolymer layer, a wellknown effect in polymer layer overstructuring. With these embedded nanostructures the polymer already bursts at $5 \mathrm{~mW}$ illumination and shows topographic deformations 
already for $0.2 \mathrm{~mW}$. At this small power density, small blisters appeared around the nanotriangles. However, they appeared not only along the boundaries of the triangles, but were distributed over the whole viewing field (Fig. 6.7). Due to these topographical features, phase contrasts in the order of about $20^{\circ}$ appeared which superpose possible phase contrasts in the order of $1^{\circ}$ which could stem from crosslinking. In this case, clear detection of photochemical induced decay and crosslinking is also not possible.

\subsection{Photochemical experiment with copolymers carrying protected amines}

After these experiments using photolabile copolymers we now concentrate on copolymers with protected amine side groups. Protected amine groups have a large variety of applications in synthesis [30] and in surface patterning [2, 3, 31, 32]. Photochemistry allows for selective deprotection of the amines and subsequent binding of many different kinds of molecules or particles. For our purposes, we synthesized a copolymer P(MMA-co-Nvoc-AEMA) with RAFT consisting of 2/3 MMA and 1/3 aminoethylmethacrylate (AEMA). The AEMA is protected by nitroveratryloxycarbonyl (NVOC) groups (Fig. 6.2 (c)). The photoreaction causes removal of the NVOC groups; the deprotected amines are then accessible to bind to different kinds of molecules, such as fluorescence markers to label the reaction volume as shown here for fluorescein (Fig. 6.8). Monitoring with sub-wavelength resolution is then possible using specialized superresolution fluorescence microscopy techniques such as stochastic optical reconstruction microscopy (STORM) [33, 34], stimulated emission depletion (STED) microscopy $[35,36]$ or photoactivated localization microscopy (PALM) [37, 38]; notably these techniques were honored with the 2014 Nobel Prize in Chemistry.

The block copolymer was dissolved in MEK and spin-coated over glass and LNO surfaces resulting in dense polymer layers with a now reduced thickness of $30 \mathrm{~nm}$. After writing lines at different power densities, all samples were immersed into a solution containing fluorescein isothiocyanate (FITC) molecules in order to fluorescently label the deprotected amine groups (Fig. 6.8). The written lines became visible in fluorescence images for power densities around 1-2 mW (Fig. 6.9(a)). Similar to the abovereported experiments, no difference was found for this copolymer when using glass and LNO substrates, and no photoreaction took place under cw illumination. Again, two-photon absorption turns out to be the dominant reaction mechanism here. Besides the lines, a homogeneous background of non-specifically bound fluorophores, which could not washed away, appeared over the whole area.

The same experiment carried out with $10 \mathrm{~nm}$ high Fischer patterns on the glass substrate is shown in (Fig. 6.9 (b)). In comparison to the pure glass surface, the photoreaction takes place already for a power threshold around $0.5 \mathrm{~mW}$ (as opposed to the 1-2 $\mathrm{mW}$ before) indicating that the particle near-fields increased the photoreaction. 

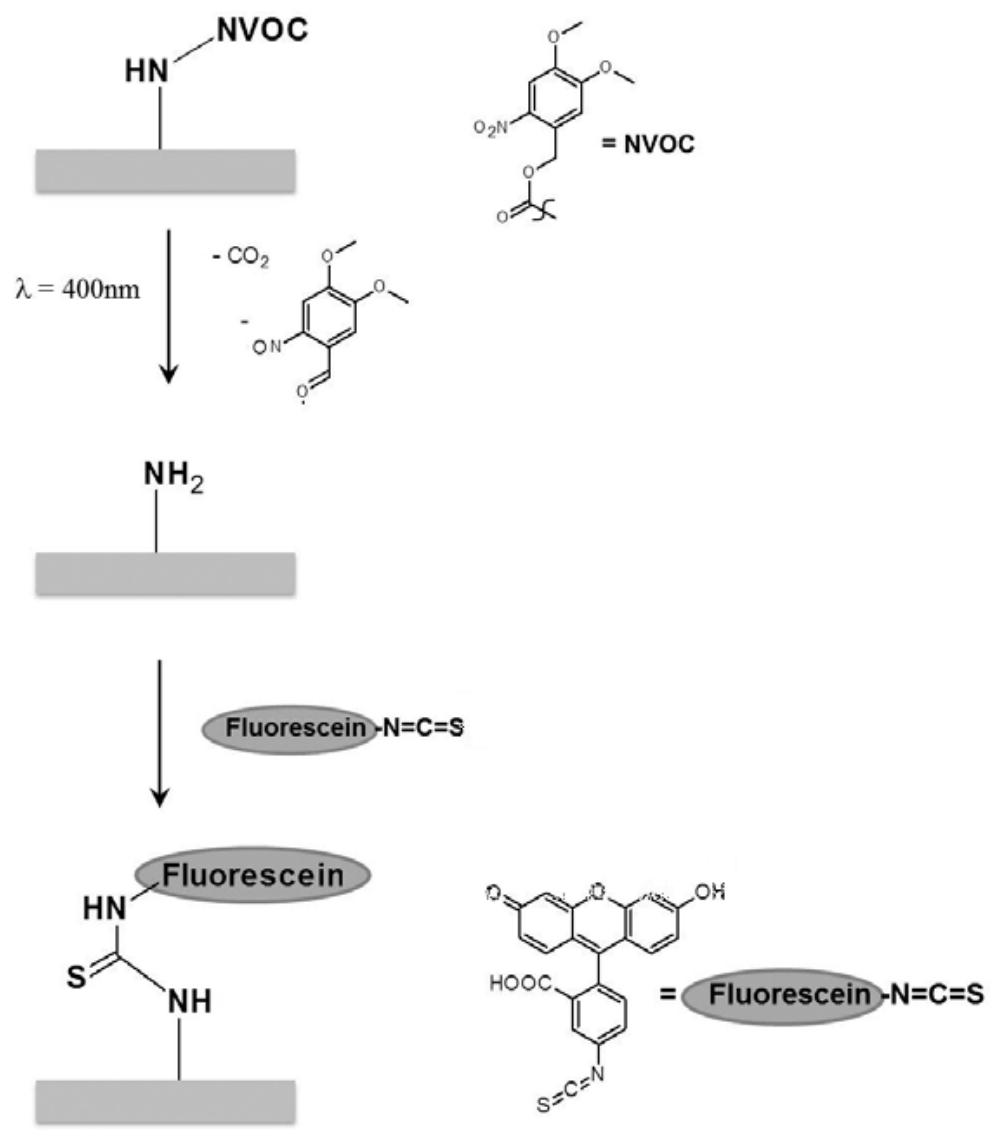

Fig. 6.8: Sketch of the chemical method of deprotecting the NVOC functionalized amines and subsequently binding the fluorescein (FITC) molecules. Note that the same route can also be applied successfully with a variety of other markers.

However, the confocal fluorescence microscope used for monitoring so far is not able to provide any sub-wavelength resolution. For that reason we investigated the same kind of samples by STORM [33,34], a method which allows imaging of the bound fluorescence markers with a resolution well below the $100 \mathrm{~nm}$ threshold. The more stable fluorescence marker Alexa 647 was used for the STORM measurements. After photochemical writing, the samples were immersed for $2 \mathrm{~h}$ in a solution containing the marker in methanol. Note that the methanol causes a slight swelling of the polymer matrix so that the marker molecules may also penetrate the whole polymer matrix and non-specifically bind to the reaction volumes in the neighborhood of the gold triangles inside the matrix, not only to their surface. This penetration is facilitated by the reduced layer thickness of $30 \mathrm{~nm}$. As the Fischer patterns are buried under the polymer matrix, their near-fields and the resulting photoreaction volumes are mainly 

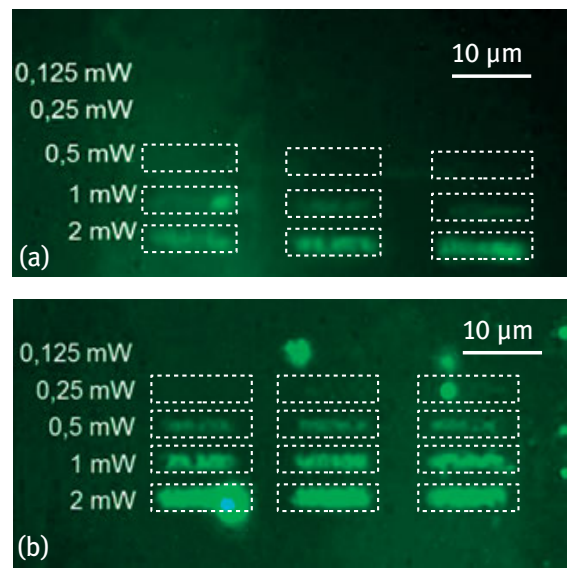

Fig. 6.9: Confocal fluorescence images of lines photochemically written into the $70 \mathrm{~nm}$ thick copolymer layer with protected amine side groups (a) on pure glass and (b) on glass carrying a $10 \mathrm{~nm}$ thick Au Fischer pattern. The deprotected amines were labeled with FITC (Fig. 6.8) after photochemical writing in order to render the free amine side groups visible by confocal fluorescence microscopy. As shown, the Fischer-patterns considerably enhance the photochemical processing.
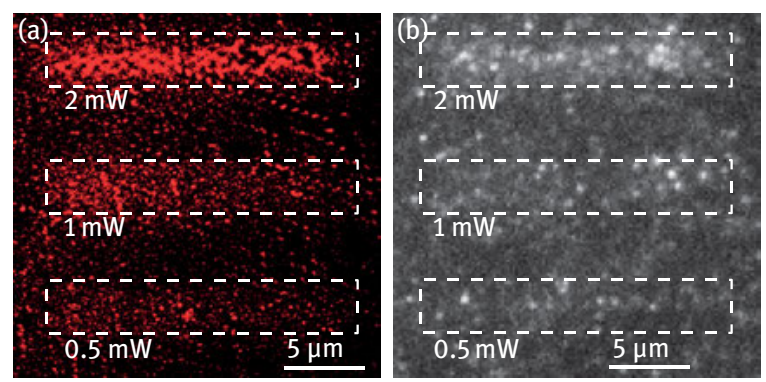

Fig. 6.10: STORM images of Alexa 647 fluorescence markers attached to amine side groups following the same recipe illustrated in Fig. 6.8 for FITC attachment. Amines were deprotected photochemically by writing lines at different power densities into the $30 \mathrm{~nm}$ thick copolymer layer sitting on glass with a $10 \mathrm{~nm}$ high Fischer pattern. (a) Evaluated STORM image exhibiting a finer structure, indicating the influence of the periodic Fischer patterns on the photoreaction In contrast, (b) shows a single fluorescence image from the raw data. The written lines are marked by dashed boxes. Again, the Fischer patterns considerably enhance the photochemical processes.

located in the interior of the polymer matrix, well below its surface. After incubation with markers, the samples were immersed in pure methanol for $1 \mathrm{~h}$ in order to wash off any non-bound residuals.

The STORM image (Fig. 6.10 (a)) and a corresponding single fluorescence image (Fig. 6.10 (b)) shows a photoreaction at powers of the order of $1 \mathrm{~mW}$ in the shape of stripes of increased fluorophore density. Periodic substructures of these stripes with a periodicity below $1 \mu \mathrm{m}$ correspond to periodic Fischer pattern structures. They 
clearly indicate the influence of the nanoparticles' near-field distribution on the local photoreactions. Further experiments trying to increase the quality and resolution of these images are in progress. In this image, the lines are also accompanied by non-specifically bound fluorophores around the written lines.

\subsection{Conclusions}

We triggered nonlinear optically induced photoreactions in several MMA-based copolymers using three different photolabile groups. We focused on proving photochemical reactions in the near-field of metallic nanoparticles embedded in the polymer matrix. Basic tests were carried out in order to demonstrate whether or not such photoreactions may be selectively triggered through second harmonic generation (SHG) from nanoparticles as opposed to multiphoton absorption processes. Our experiments showed that direct two-photon absorption is the dominant photoreaction mechanism in all cases.

Nevertheless, good localization and enhancement of the photoreaction in the near-fields of metallic nanoparticles can always be expected. However, for the azobenzenes and azosulfonates, no localized photoreactions were found, as the azobenzene reaction only changes absorbance, and the elasticity change due to azosulfonate decay is superposed to topographic changes due to side reactions in the polymer thin film. The only promising way of proving such near-field enhanced reactions turned out to be the deprotection of NVOC-protected amines which in turn allowed labeling of the reaction volume for high-resolving fluorescence microscopy. In principle, the advantage of this deprotection reaction is the wide variety of molecules (or even nanostructures) which exist and are ready to bind site-specifically to the deprotected amines for further applications. This type of photoactive unit has now been implemented into the membrane of polymersomes, a class of artificial vesicles. Photochemical experiments using near-field effects with integrated gold nanoparticles at those polymersomes are in progress. A site-specific modification of the polymersomes induced by a biologically harmless irradiation is envisioned.

\section{Acknowledgments}

P. R., U. G. and T. K. acknowledge financial support from the German Science Foundation (DFG) through the DFG priority program SPP 1327 and the Cluster of Excellence "Center of Advancing Electronics Dresden", as well as support from the European Social Funds through the ESF-NFG project "Mind Nano". 


\section{References}

[1] Levinson HJ, Arnold WH. Optical Lithography. In: Rai-Choudhury P, ed. Handbook of Microlithography, Micromachining, and Microfabrication. Bellingham, USA, SPIE Press, 1997.

[2] Braun F, Eng L, Trogisch S, Voit B. Novel labile protected amine terpolymers for the preparation of patterned functionalized surfaces: Synthesis and characterization. Macromol. Chem. Phys. 2003, 204, 1486-1496.

[3] Opitz J, Braun F, Seidel R, Pompe W, Voit B, Mertig M. Site-specific binding and stretching of DNA molecules at UV-light-patterned aminoterpolymer films. Nanotech. 2004, 15, 717-723.

[4] Deeb C, Zhou X, Gerard D et al. Off-resonant optical excitation of gold nanorods: Nanoscale imprint of polarization surface charge distribution. J. Phys. Chem. Lett. 2011, 2, 7-11.

[5] Wang L, Uppuluri SM, Jin EX, Xu X. Nanolithography using high transmission nanoscale bowtie apertures Nano Lett. 2006, 6, 361-364.

[6] Ueno K, Juodkazis S, Shibuya T et al. Nanoparticle plasmon-assisted two-photon polymerization induced by incoherent excitation source. J. Am. Chem. Soc. 2008, 130, 6928-6929.

[7] Murazawa N, Ueno K, Mizeikis V, Juodkazis S, Misawa H. Spatially selective nonlinear photopolymerization induced by the near-field of surface plasmons localized on rectangular gold nanorods. J. Phys. Chem. C 2009, 113, 1147-1149.

[8] Sundaramurthy A, Schuck PJ, Conley NR, Fromm DP, Kino GS, Moerner WE. Toward NanometerScale optical photolithography: Utilizing the Near-Field of bowtie optical nanoantennas. Nano Lett. 2006, 6, 355-360.

[9] Reichenbach P, Horneber A, Gollmer D et al. Nonlinear-optical point light sources through field enhancement at metallic nanocones. Opt. Expr. 2014, 22, 15484-15501.

[10] Reichenbach P. Metallische Nanoantennen: Frequenzverdopplung und photochemische Reaktionen auf kleinen Skalen. PhD thesis. Dresden, Germany, Technische Universität Dresden, 2012.

[11] Georgi U. Synthese photoreaktiver Polymere zur optischen Strukturierung dünner Schichten. PhD thesis. Dresden, Germany, Technische Universität Dresden, 2014.

[12] Georgi U, Reichenbach P, Oertel U, Eng LM, Voit B. Synthesis of azobenzene-containing polymers and investigation of their substituent-dependent isomerisation behaviour. Reac. Funct. Polymer 2012, 72, 242-251.

[13] Aussenegg FR, Leitner A, Gold H. Optical second-harmonic generation of metal-island films. Appl. Phys. A 1995, 60, 97-101.

[14] Bozhevolnyi SI, Beermann J, Coello V. Direct observation of localized second-harmonic enhancement in random metal nanostructures. Phys. Rev. Lett. 2003, 90, 197403-1-4.

[15] Danckwerts M, Novotny L. Optical frequency mixing at coupled gold nanoparticles. Phys. Rev. Lett. 2007, 98, 026104-1-4.

[16] Lesuffleur A, Kumar LKS, Gordon R. Enhanced second harmonic generation from nanoscale double-hole arrays in a gold film. Appl. Phys. Lett. 2006, 88, 261104-1-3.

[17] Eftekhari F, Gordon R. Enhanced second harmonic generation from noncentrosymmetric nanohole arrays in a gold film. IEEE J. Sel. Top. in Quant. 2008, 14, 1552-1558.

[18] Reichenbach P, Eng LM, Georgi U, Voit B. 3D-steering and superfocusing of second-harmonic radiation through plasmonic nano antenna arrays. J. Laser Appl. 2012, 24, 042005-1-8.

[19] Braun F, Eng LM, Loppacher C, Trogisch S, Voit B: Novel Diazosulfonate terpolymers for the preparation of structured functionalized surfaces: Synthesis and characterization. Macromol. Chem. Phys. 2002, 203, 1781-1789.

[20] Fischer UC, Zingsheim H. Submicroscopic pattern replication with visible light. J. Vac. Sci. Technol. 1981, 19, 881-885. 
[21] Kosiorek A, Kandulski W, Chudzinski P, Kempa K, Giersig M. Shadow nanosphere lithography: simulation and experiment. Nano Lett. 2004, 4, 1359-363.

[22] Li H, Low J, Brown KS, Wu N. Large-area well-ordered nanodot array pattern fabricated with self-assembled nanosphere template. IEEE Sens. J. 2008, 8, 880-884.

[23] Nuyken O, Scherer C, Baindl A, Brenner AR, Dahn U. Azo-group-containing polymers for use in communications technologies. Prog. Polym. Sci. 1997, 22, 93-183.

[24] Hvilsted S, Sanchez C, Alcala R. The volume holographic optical storage potential in azobenzene containing polymers. J. Mater. Chem. 2009, 19, 6641-6648.

[25] Chiefari J, Chong YK, Ercole F et al. Living Free-Radical Polymerization by Reversible Addition Fragmentation Chain Transfer: The RAFT Process. Macromolecules 1998, 31, 5559-5562.

[26] Feldermann A, Ah Toy A, Phan H, Stenzel MH, Davis TP, Barner-Kowollik C. Reversible addition fragmentation chain transfer copolymerization: influence of the RAFT process on the copolymer composition, Polymer 2004, 45, 3997-4007.

[27] Favier A, Charreyre MT, Experimental Requirements for an Efficient Control of Free-Radical Polymerizations via the Reversible Addition-Fragmentation Chain Transfer (RAFT) Process. Macromol. Rapid Commun. 2006, 27, 653-692.

[28] Nuyken O, Voit B. Water-soluble and photosensitive copolymers from methyl methacrylate and sodium 3-vinylphenylazosulfonate. Makromol. Chem. 1989, 190, 1325-1332.

[29] Nuyken 0, Voit B. The photoactive diazosulfonate group and its role in polymer chemistry. Makromol. Chem. Phys. 1997, 198, 2337-2372.

[30] Pillai VNR. Photoremovable protecting groups in organic synthesis. Synthesis 1980, 1, 1-26.

[31] Millaruelo M, Eng LM, Mertig M et al. Photolabile Carboxylic Acid Protected Terpolymers for Surface Patterning. Part 2: Photocleavage and Film Patterning, Langmuir 2006, 22, 9446-9452.

[32] Sieczkowska B, Millaruelo M, Messerschmidt M, Voit B. New Photolabile Functional Polymers for Patterning onto Gold Obtained by Click Chemistry. Macromolecules 2007, 40, 2361-2370.

[33] Rust MJ, Bates M, Zhuang X. Sub-diffraction-limit imaging by stochastic optical reconstruction microscopy (STORM). Nature Methods 2006, 3, 793-796.

[34] Hess ST, Girirajan TPK, Mason MD. Ultra-High Resolution Imaging by Fluorescence Photoactivation Localization Microscopy. Biophys. J. 2006, 91, 4258-4272.

[35] Hell SW, Wichmann J. Breaking the diffraction resolution limit by stimulated emission: stimulated-emission-depletion fluorescence microscopy. Opt. Lett. 1994, 18, 780-782.

[36] Klar TA, Hell SW. Subdiffraction resolution in far-field fluorescence microscopy. Opt. Lett. 1999, 24, 954-956.

[37] Betzig E. Proposed Method for molecular optical imaging, Opt. Lett. 1995, 20, 237-239.

[38] Betzig E, Patterson GH, Sougrat R et al. Imaging Intracellular Fluorescent Proteins at Nanometer Resolution. Science 2006, 313, 1642-1645. 
